\title{
Deep learning-based quantification of arbuscular mycorrhizal fungi in plant roots
}

\author{
Edouard Evangelisti $^{1}$ (D), Carl Turner ${ }^{2}$ (D), Alice McDowell ${ }^{1}$, Liron Shenhav ${ }^{1}$ (D), Temur Yunusov ${ }^{1}$ (D), \\ Aleksandr Gavrin ${ }^{1}$ iD, Emily K. Servante ${ }^{3}$, Clément Quan ${ }^{1}$ iD and Sebastian Schornack ${ }^{1}$

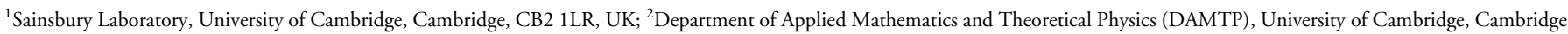 \\ CB3 0WA, UK; ${ }^{3}$ Department of Plant Sciences, University of Cambridge, Cambridge, CB2 3EA, UK
}

Authors for correspondence:

Edouard Evangelisti

Email: edouard.evangelisti@slcu.cam.ac.uk

Sebastian Schornack

Email: sebastian.schornack@s/cu.cam.ac.uk

Received: 3 July 2021

Accepted: 17 August 2021

New Phytologist (2021)

doi: $10.1111 / \mathrm{nph} .17697$

Key words: classification, ClearSee, ConvNet, image analysis, mycorrhiza, Rhizophagus, root.

\section{Summary}

- Soil fungi establish mutualistic interactions with the roots of most vascular land plants. Arbuscular mycorrhizal (AM) fungi are among the most extensively characterised mycobionts to date. Current approaches to quantifying the extent of root colonisation and the abundance of hyphal structures in mutant roots rely on staining and human scoring involving simple yet repetitive tasks which are prone to variation between experimenters.

- We developed Automatic Mycorrhiza Finder (AMFinder) which allows for automatic computer vision-based identification and quantification of AM fungal colonisation and intraradical hyphal structures on ink-stained root images using convolutional neural networks.

- AMFinder delivered high-confidence predictions on image datasets of roots of multiple plant hosts (Nicotiana benthamiana, Medicago truncatula, Lotus japonicus, Oryza sativa) and captured the altered colonisation in ram1-1, str, and smax1 mutants. A streamlined protocol for sample preparation and imaging allowed us to quantify mycobionts from the genera Rhizophagus, Claroideoglomus, Rhizoglomus and Funneliformis via flatbed scanning or digital microscopy, including dynamic increases in colonisation in whole root systems over time.

- AMFinder adapts to a wide array of experimental conditions. It enables accurate, reproducible analyses of plant root systems and will support better documentation of AM fungal colonisation analyses. AMFinder can be accessed at https://github.com/SchornacklabSLCU/ amfinder.

\section{Introduction}

Soil fungi establish mutualistic interactions with the roots of $>85 \%$ of vascular land plants (Brundrett \& Tedersoo, 2018). These interactions, termed mycorrhizas, lead either to the formation of a dense hyphal sheath surrounding the root surface (ectomycorrhizas) or to fungal hyphae penetrating host tissues (endomycorrhizas) (Brundrett, 2007). The best-characterized type of endomycorrhiza, called arbuscular mycorrhiza (AM), involves species from the subphylum Glomeromycotina (Schüßler et al., 2001; Spatafora et al., 2016). Arbuscular mycorrhizal fungal hyphae grow toward plant roots following the exchange of diffusible chemical cues (Luginbuehl \& Oldroyd, 2017). At root surface penetration points, hyphae differentiate into swollen or branched structures termed hyphopodia. Following entry and crossing of the root epidermis, hyphae spread between cortical cells (Arum-type colonization) or via intracellular passages of cortical cells (Paris-type colonization)
(Dickson, 2004). The differentiation of highly branched intracellular exchange structures, the arbuscules, accompanies hyphal growth and enables a reciprocal transfer of nutrients between symbionts (Luginbuehl \& Oldroyd, 2017). Postarbuscular development includes the differentiation of vesicles and spores. While these successive differentiation events reflect a precise morphogenetic program, the whole hyphal network is not synchronized. As a result, the various types of intraradical hyphal structures occur simultaneously inside plant roots (Montero et al., 2019).

Rhizophagus irregularis (formerly Glomus intraradices) is one of the most extensively characterised mycobionts in endomycorrhiza research. To date, genetic manipulation of $R$. irregularis remains challenging (Helber \& Requena, 2008) and the main advances in AM fungal symbiosis research relate to the experimentally more tractable plant hosts. The extent of root colonisation and the relative abundance of intraradical hyphal structures in mutant roots are essential parameters for characterising host genes that underlie 
mycorrhiza establishment and accommodation (Montero et al., 2019). Mycorrhiza-responsive host genes facilitate the molecular quantification of fungal colonisation. For instance, expression of the Medicago truncatula Phosphate transporter 4 (MtPT4) gene is limited to the root tip in the absence of mycorrhiza (Volpe et al., 2016), while cells with arbuscules express MtPT4 to enable plant acquisition of inorganic phosphate (Harrison et al., 2002; Maeda et al., 2006; Javot et al., 2007). Likewise, the abundance of transcripts encoding $M$. truncatula Blue Copper-Binding Protein 1 and Lotus japonicus apoplastic subtilase SbtM correlate with stage transitions during arbuscule development (Hohnjec et al., 2005; Takeda et al., 2009; Parádi et al., 2010). Complementary to molecular methods and independent of gene sequence knowledge is the visual diagnosis of AM fungal colonisation. It involves differential staining of fungal cell walls (Vierheilig et al., 1998, 2005; Hulse, 2018) followed by random sampling and counting using a grid-intersect method (Giovannetti \& Mosse, 1980). This method is considered a standard in mycorrhiza research (Sun \& Tang, 2012).

Deep learning encompasses an extensive class of computational models that learn to extract information from raw data at multiple levels of abstraction, thereby mimicking how the human brain perceives and understands information (Voulodimos et al., 2018). In supervised learning problems, where example data labelled with correct outputs are available, these models can be iteratively improved to minimise discrepancies between correct and model-predicted outputs considering all possible interfering factors (O'Mahony et al., 2020). With the increase in computing power over recent years, deep learning has fostered tremendous data analysis advances. Computer vision is one of the most iconic examples, with the development of convolutional neural networks (CNNs), a class of deep learning methods inspired by models of the visual system's structure (LeCun et al., 1998). A basic image classification CNN begins with a stack of convolutional and pooling layers, each providing the input to the next; these allow detection of increasingly large and complex features in the input image whilst preserving the grid-like nature of the input (Voulodimos et al., 2018; Dhillon \& Verma, 2020). This is followed by one or more fully connected layers of neurons which take the resulting feature map and implement the highlevel reasoning needed to classify the image. Convolutional neural networks underlie breakthrough advances in diverse technological and biomedical domains including face recognition, object detection, diagnostic imaging, and self-driving cars (Matsugu et al., 2003; Szarvas et al., 2005; Bojarski et al., 2016; Yamashita et al., 2018). Convolutional neural networks also benefit botany by enabling the identification of flowers (Liu et al., 2017) and ornamental plants (Sun et al., 2017), while CNNbased plant pathology diagnostic tools identify crop diseases based on leaf symptoms (Mohanty et al., 2016; Ferentinos, 2018; Thapa et al., 2020).

We took advantage of CNNs to develop the Automatic Mycorrhiza Finder (AMFinder), an automatic, user-supervised tool suite for in silico analysis of AM fungal colonisation and recognition of intraradical hyphal structures. Using AMFinder, we quantified fungal colonisation dynamics on whole Nicotiana benthamiana root systems using low-resolution, flatbed scanneracquired images of ink-stained roots. AMFinder accurately quantified changes in the extent of $R$. irregularis colonisation in M. truncatula ram1-1, str, and Oryza sativa smax1 plant mutants compared to their wild-type lines. Moreover, AMFinder robustly identified colonised root sections and intraradical hyphal structures in several plant species commonly used in mycorrhiza research, including $M$. truncatula, L. japonicus, and O. sativa, and is compatible with the AM fungi Claroideoglomus claroideum, Rhizoglomus microaggregatum, Funneliformis geosporum and Funneliformis mosseae. We developed a command-line tool paired with a standalone graphical browser to enable efficient browsing of large images and manual curation of computer predictions. Overall, our work provides a framework for reproducible automated phenotyping of AM fungal colonisation of plant roots.

\section{Materials and Methods}

\section{Plant material}

The $N$. benthamiana line used here is a laboratory cultivar obtained from The Sainsbury Laboratory, Norwich, UK, originating from Australia (Bally et al., 2018). M. truncatula R108 seeds were provided by Giles Oldroyd (The Sainsbury Laboratory). L. japonicus cv Gifu seeds were provided by Simona Radutoiu (Aarhus University, Denmark). The rice (O. sativa subsp. japonica) plant material has been described elsewhere (Choi et al., 2020).

\section{Seed germination}

Nicotiana benthamiana seeds were germinated on Levington F2 compost (ICL, Ipswich, UK) for $1 \mathrm{wk}$ at $24^{\circ} \mathrm{C}$ with a $16 \mathrm{~h}: 18$ h, light : dark photoperiod. $M$. truncatula seeds were scarified in sulphuric acid for $5 \mathrm{~min}$, rinsed in sterile water and surfacesterilized in bleach for $5 \mathrm{~min}$. Seeds were then soaked in water for $30 \mathrm{~min}$ and stratified for $3 \mathrm{~d}$ at $4^{\circ} \mathrm{C}$ in the dark. L. japonicus seeds were scarified with sandpaper, surface-sterilized in bleach for $15 \mathrm{~min}$ and soaked overnight in water at $4^{\circ} \mathrm{C}$. Germination was induced at $20^{\circ} \mathrm{C}$. O. sativa seed germination was described elsewhere (Choi et al., 2020).

\section{Growth conditions for arbuscular mycorrhizal colonisation}

One-week-old seedlings were transferred to $6 \times 5$ cellular trays containing silver sand supplemented with a $1: 10$ volume of AM fungal crude inoculum and grown at $24^{\circ} \mathrm{C}$ with a $16 \mathrm{~h}: 8 \mathrm{~h}$, light : dark photoperiod. $R$. irregularis, $C$. claroideum and F. geosporum crude inocula were obtained from PlantWorks (Sittingbourne, UK). Funneliformis mosseae crude inoculum was obtained from MycAgro (Bretenière, France). N. benthamiana plants were watered with a low-phosphate Long Ashton nutrient solution (Hewitt, 1966), while milliQ (Merck Millipore, Watford, UK) water was used for L. japonicus and M. truncatula plants. O. sativa growth conditions and AM colonisation conditions were described elsewhere (Choi et al., 2020). Plant roots 
were harvested at either 4 or 6 wk post-inoculation (wpi) and directly used for staining or total mRNA extraction.

\section{Fungal staining}

A modified ink-vinegar method (Vierheilig et al., 1998) was used to stain fungal structures within plant roots. Briefly, roots from 4- and 6-wk-old plants were incubated in 10\% (w/v) potassium hydroxide $(\mathrm{KOH})$ for $10 \mathrm{~min}$ at $95^{\circ} \mathrm{C}$ and rinsed in $5 \%(\mathrm{v} / \mathrm{v})$ acetic acid before staining in staining solution $(5 \%(\mathrm{v} / \mathrm{v})$ Sheaffer Skrip black ink, 5\% (v/v) acetic acid) (A. T. Cross Co., Providence, RI, USA) for $10 \mathrm{~min}$ at $95^{\circ} \mathrm{C}$. Stained roots were rinsed in distilled water, followed by clearing in ClearSee (Kurihara et al., 2015) for 1 min (Supporting Information Fig. S1). Cleared roots were mounted in a glycerol-containing mounting medium (20\% (v/v) glycerol, $50 \mathrm{mM}$ Tris- $\mathrm{HCl} \mathrm{pH}$ 7.5, $0.1 \%$ (v/v) Tween-20).

\section{Scanning and bright field imaging}

Low-magnification images of ink-stained roots were acquired with an Epson Perfection flatbed scanner (Epson UK, Hemel Hempstead, UK) using default settings and a resolution of 3200 dots per inch (Fig. S2). High-magnification images were acquired with a VHX-5000 digital microscope (Keyence, Milton Keynes, UK) equipped with a $\times 50-200$ zoom lens set to $\times 200$ magnification, using transillumination mode and focus stacking. Images of $M$. truncatula arbuscules were obtained with an Axio Imager M2 (Zeiss) microscope equipped with a $\times 64$ numerical aperture (NA) 1.4 oil immersion objective lens using differential interference contrast illumination.

\section{Quantitative reverse-transcription polymerase chain reaction (qRT-PCR)}

Total RNA was extracted from $100 \mathrm{mg}$ root material using an RNeasy Mini Kit (Qiagen) according to the manufacturer's instructions. Quality was assessed by electrophoresis on an agarose gel. One microgram was reverse transcribed to generate first-strand complementary DNA (cDNA) using an iScript cDNA Synthesis Kit (Bio-Rad). Quantitative reversetranscription polymerase chain reaction experiments were performed with $2.5 \mu \mathrm{l}$ of a $1: 20$ dilution of first-strand cDNA and LightCycler 480 SYBR Green I Master mix, according to the manufacturer's instructions (Roche). Gene-specific oligonucleotides were designed using the BATCHPRIMER3 software (You et al., 2008), and their specificity was validated by analysing dissociation curves after each run. Genes encoding L23 (Niben101Scf01444g02009) and FBOX (Niben101Scf04495g02005) were selected as constitutive internal controls for $N$. benthamiana (Liu et al., 2012). Primers for transcripts encoding RiEF1a and NbBCP1b (Niben101Scf07438g04015.1) are listed in Table S1. Six biological replicates of the entire experiment were performed. Gene expression was normalised with respect to constitutively expressed internal controls (Vandesompele et al., 2002) and plotted using R (https://www.r-project.org/).

\section{Software design}

Implementation AMFinder implements a semi-automatic pipeline for quantification of fungal colonisation and intraradical hyphal structures in root pictures. It comprises a command-line program (amf) for root image analysis and a standalone interface (amfbrowser) for user supervision and validation of amf predictions (Fig. 1). amf is written in PYTHON (https://www.python.org/) (van Rossum \& Drake, 2009; Srinath, 2017) and uses the widely used Tensorflow (https://www.tensorflow.org/) and Keras (https://keras.io/) machine learning libraries (Chollet, 2015; Abadi et al., 2016). amfbrowser is written in OCAML (https://oca $\mathrm{ml.org} /$ ) (Leroy et al., 2020) using the 2D graphics library CAIRO (https://www.cairographics.org/) and the cross-platform widget toolkit GTK (https://www.gtk.org/). Both programs communicate through a standard ZIP archive file that stores amf probabilities, user annotations, and image settings. AMFinder deploys on Microsoft Windows, MACOS and GNU/LINUX.

Analysis pipeline The AMFinder pipeline consists of three or five steps depending on image resolution. First, image segmentation is performed by amf during an initial pre-processing step. Images are divided into square tiles using a user-defined tile size depending on image magnification and resolution (Fig. 1a). Next, tiles are analysed individually to label colonised root segments (Fig. 1a). The third step of the analysis consists of the user-supervised conversion of amf predictions (i.e. probabilities) to annotations using amfbrowser (Fig. 1a,b). If resolution allows, a second round of amf predictions can be achieved to assess the occurrence of intraradical hyphal structures on colonised tiles only. As for the first round of predictions, computer-generated probabilities are then converted to annotations under user supervision using amfbrowser (Fig. 1a,b).

\section{Deep learning}

Classifier design Identifying AM fungal colonisation and intraradical hyphal structures on images of stained roots constitutes a computer vision problem that is efficiently solved by CNNs. AMFinder implements two independent CNN-based classifiers to predict colonisation (CNN1) and intraradical hyphal structures (CNN2) (Fig. S3). As the features we are interested in are mainly small, local structures, $\mathrm{CNN} 1$ was chosen to be a fairly shallow network. It comprises four blocks of $3 \times 3$ filters (convolutions) interleaved with size-reduction layers (maximum pooling) (Fig. S3a), followed by a classifier made of three fully connected layers which compute the probabilities of each tile belonging to the mutually exclusive classes 'colonised root section' $(\mathrm{M}+)$, 'noncolonised root section' (M-), and 'background/ not a root/other' (Figs 1a, S3b).

The CNN2 classifier predicts the occurrence of arbuscules (A), vesicles $(\mathrm{V})$, hyphopodia $(\mathrm{H})$, and intraradical hyphae (IH) on tiles labeled as 'colonised root section' (M+) during $\mathrm{CNN} 1$ analysis. Its architecture is essentially the same as that of CNN1 (Fig. S3a). However, the probability that each different type of intraradical hyphal structure is present is 
(a)
Acquisition

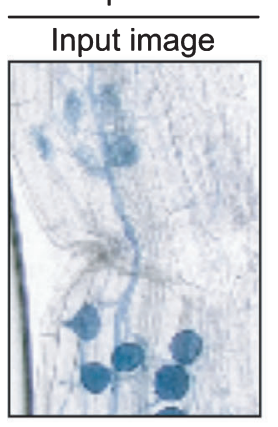

amf Preprocessing Tile mosaic
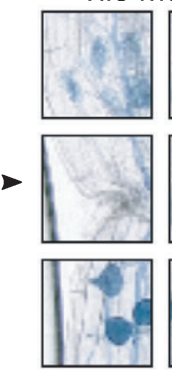

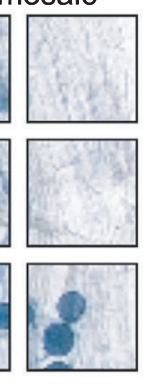

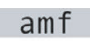

f

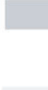

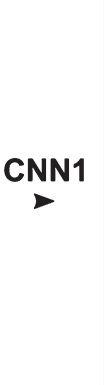

amfbrowser

Root colonisation

(b)

\begin{abstract}
PREDICTION STAGE
\end{abstract}
(1) CNN1. Root colonisation

ACTIVE TILE

(2)
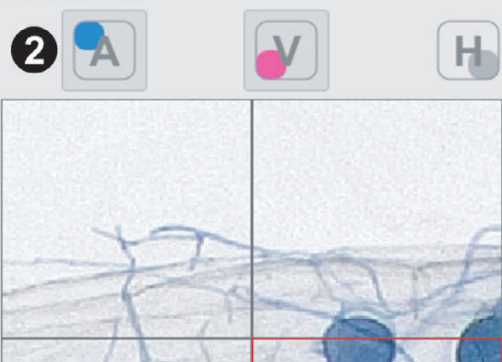

H $\mathrm{IH}$

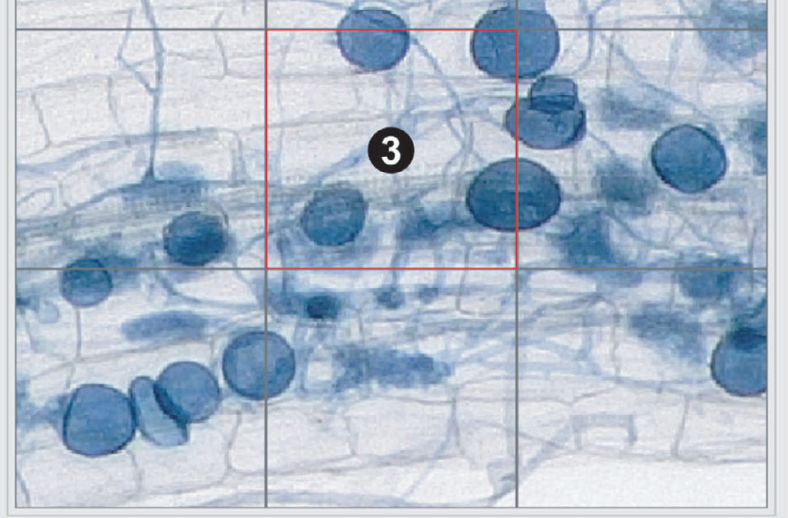

amfbrowser 2.0
Colonised

root section

Non-colonised root section

Other amf

amfbrowser

AM fungal structures

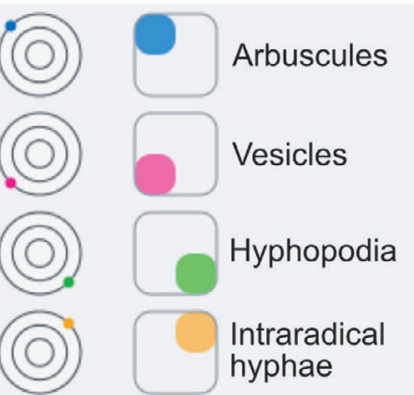

Fig. 1 AMFinder enables a semi-automated, user-supervised analysis of arbuscular mycorrhizal fungal colonisation in silico. (a) AMFinder uses a two-stage prediction pipeline for image annotation. First, input images are split into tiles and processed by amf neural network 1 (CNN1) to identify colonised root sections. Computer predictions are displayed as pie charts and are converted to annotations under user supervision. If resolution allows, tiles corresponding to colonised areas can be further analysed by amf CNN2 to identify intraradical hyphal structures. The probabilities of occurrence of the different structures are shown in a radar plot. (b) Representative screenshot of an amfbrowser annotation session. (1) Buttons to switch the display between prediction stages. (2) Clickable buttons to define the annotations present in the active tile. (3) Magnified view of the active tile (red square) and eight surrounding tiles. (4) Annotation mosaic overview. (5) Layer toolbar to filter the display. Numbers indicate annotation counts for the whole image. (6) Prediction toolbar to load predictions, fix ambiguous cases and generate annotations. (7) Export functions.

computed by a separate stack of three fully connected layers atop the convolutional and pooling layers (Fig. S3c). This design was chosen to facilitate future extensions of AMFinder, for instance to predict other types of structures (e.g. spores) or variations of a given structure (e.g. different degrees of arbuscule branching), by allowing additional expressive power to be quickly added to the network.

Datasets The CNN1 training dataset comprised 32 images of ink-stained, ClearSee-treated N. benthamiana roots colonised with $R$. irregularis, acquired using either a flatbed scanner (five images) or a digital microscope (27 images), yielding 175105 tiles annotated as follows: 15364 tiles belonged to the 'colonised root section' annotation class, 19455 tiles corresponded to noncolonised roots, and 140286 tiles consisted of background, small debris, and air bubbles (Table S2).

The CNN2 training dataset consisted of 55 high-resolution images of colonised $N$. benthamiana roots prepared as described in the earlier section. It comprised 20 564, 16 420, and 25077 tiles containing arbuscules, vesicles and intraradical hyphae, 
respectively. Only 508 tiles contained hyphopodia, preventing us from achieving efficient hyphopodia training due to the scarcity of this hyphal structure (Table S3).

A bootstrap method (a highly simplified type of uncertainty sampling as used in active learning) was used to annotate images. First, 10 images were manually annotated through amfbrowser and used to train a development (pre-alpha) version of AMFinder CNNs. Trained CNNs were then used to annotate the remaining images. Computer predictions were individually inspected through amfbrowser to correct certain low-confidence predictions (namely those with all network outputs $<0.5$ ).

Datasets were randomly split into two subsets for training and validation in an $85: 15$ ratio. When data augmentation was used, the tiles in the training set were augmented by randomly applying the following modifications: horizontal flips, vertical flips, brightness adjustment $( \pm 25 \%)$, colour inversion (image negation), desaturation (to create a grayscale image), or random adjustment of hue (rotating colours around a colour wheel).

An independent test set was used for independent assessment of CNN capabilities (Table S4). It consisted of 20 manually annotated images of 81 tiles each, acquired with either a flatbed scanner (10 images, used with CNN1 only) or a digital microscope (10 images, used for both CNNs).

Classifier parameters and training Both classifiers were trained for 100 epochs (i.e. complete training cycles) with a batch size of 32 during both the initial bootstrapping stage described in the previous paragraph and the main training stage. For optimal training, background over-representation was compensated for by randomly removing excess background tiles. Training weights were assigned based on tile count in each annotation class to account for any residual imbalance. Consistent with their respective output, categorical cross-entropy was used as the CNN1 loss function, while binary cross-entropy was used for each CNN2 classifier (Gordon-Rodriguez et al., 2020). To prevent overfitting, an early stopping mechanism was used to prematurely terminate training and to restore the best-performing model parameters if the loss did not decrease for 12 training cycles in a row. Both classifiers relied on the Adam optimizer (Kingma \& $\mathrm{Ba}, 2015$ ) with an initial learning rate of 0.001 , which was exponentially decayed with a factor of 0.9. In addition, learning rate was further decreased to one fifth if the loss did not decrease for two successive epochs, until it reached a minimum rate of $10^{-6}$. AMFinder training was achieved on a High-Performance Computing (HPC) system running Linux Ubuntu, using 10 cores and 20 Gb RAM.

Classifier evaluation Three parameters were determined to evaluate the classifier results. The accuracy is given by the following relation:

$$
\text { Accuracy }=\frac{\mathrm{TP}+\mathrm{TN}}{\mathrm{TP}+\mathrm{FP}+\mathrm{TN}+\mathrm{FN}}
$$

where TP (true positive rate) indicates accurate positive identifications (e.g. a tile containing intraradical hyphal structure is identified as colonised), TN (true negative rate) indicates accurate negative identifications (e.g. a tile containing root tissues only is identified correctly as noncolonised), FP (false positive rate) indicates that the observation is different but predicted as true (e.g. a noncolonised root tile is identified as colonised), and FN (false negative rate) indicates that a true observation is predicted to be different (e.g. a colonised root tile is identified as noncolonised). Accuracy counts all kinds of errors with the same costs and classifiers were thus further analysed using sensitivity and specificity:

$$
\text { Sensitivity }=\frac{\mathrm{TP}}{\mathrm{TP}+\mathrm{FN}} \text { Specificity }=\frac{\mathrm{TN}}{\mathrm{TN}+\mathrm{FP}}
$$

A high sensitivity indicates that the annotation class is correctly recognised (i.e. there are few false negatives), while high specificity indicates that a high number of tiles not labelled with a given annotation class $x$ indeed do not belong to class $x$ (i.e. there are few false positives).

\section{Results}

\section{AMFinder robustly identifies intraradical structures in AM fungi}

Convolutional neural network-based classifiers require training to recognise the desired image classes. We trained the AMFinder CNNs using images of ink-stained roots of $N$. benthamiana plants inoculated with $R$. irregularis (Fig. 2a,b; Tables 1, 2). To improve $\mathrm{CNN}$ generalisation and reduce overfitting, we independently trained CNNs with augmented data (Fig. 2c,d; Tables 1, 2). We then assessed the performance of the CNNs on an independent test dataset (Tables 1, 2, S4). CNN1 labelled colonised $(\mathrm{M}+)$ and noncolonised $(\mathrm{M}-)$ root sections, and background (Other) with an overall accuracy of $97 \%$ (Fig. 2a,b; Table 1). The classifier performed similarly on low- (Fig. 2a) and highmagnification (Fig. 2b) images. Training on augmented data $(\mathrm{CNN} 1 \mathrm{v} 2)$ reduced the overall performance of the network to $94 \%$ when evaluated on ink-stained images (Table 1). However, CNN1v2 was able to accurately predict colonisation on grayscale and inverted images (Fig 2d), suggesting network generalisation was improved compared to CNN1v1.

CNN2 classifiers accurately identified intraradical hyphal structures on high-magnification images (Figs 2b, S4; Table 2). The overall CNN2 performance was $94 \%$ but dropped to $81 \%$ when data augmentation was active (Table 2), suggesting this method did not improve CNN2 generalisation.

To gain more insights into AMFinder performance, we generated $\mathrm{CNN} 1$ confusion matrices and extracted representative sets of mispredicted tiles (Figs S4, S5). The most frequent type of CNN1 misprediction consisted of 'colonised root sections' (M+) that were incorrectly identified as 'noncolonised root sections' (M-) (Fig. S4a,b). These tiles contained low-contrasted fungal structures (Fig. S4a,c). The presence of extraradical hyphae was an additional source of mispredictions (Fig. S4a,c). We then repeated the analysis with CNN2 classifiers (Fig. S5). As implied by the sensitivity and specificity results (Table 2), most CNN2v1 

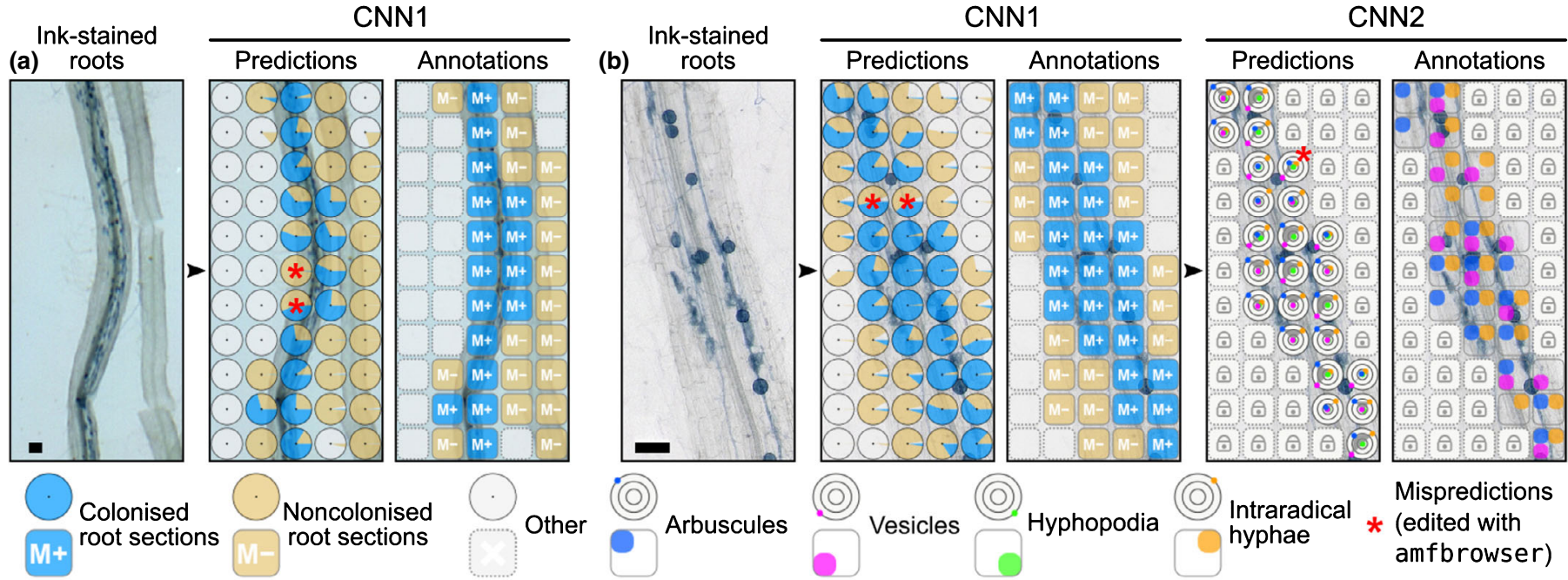

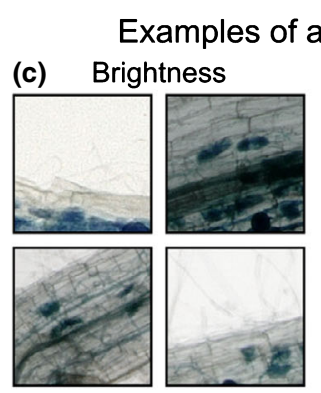

Color rotation
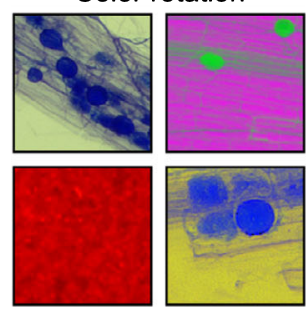

\section{ugmented tiles}

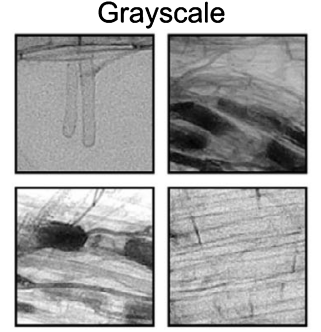

Invert

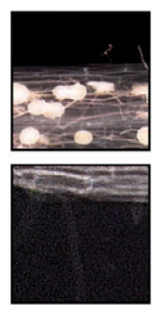

CNN1 data augmentation

(d) Input image

- +
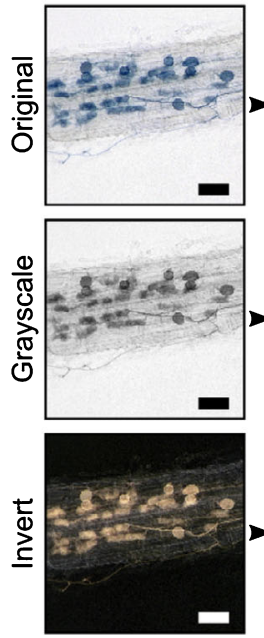

CNN2 data augmentation

Fig. 2 AMFinder accurately labels Rhizophagus irregularis colonisation and intraradical hyphal structures in Nicotiana benthamiana roots. (a) Schematic view of neural network 1 (CNN1) predictions on a low-resolution image of ink-stained $N$. benthamiana roots acquired with a flatbed scanner. (b) Schematic view of CNN1 (left) and CNN2 (right) predictions on a high-resolution image of ink-stained $N$. benthamiana roots acquired with a digital microscope. (c) Examples of augmented tiles used for CNN1 and CNN2 training. (d) Comparison of CNN1 and CNN2 performance on images of inkstained $N$. benthamiana roots colonised with $R$. irregularis, either unaltered, desaturated (grayscale), or inverted. Mispredictions (red asterisks) were manually corrected using amfbrowser. Arrowheads indicate the direction of AMFinder processing. Bars: (a) $100 \mu \mathrm{m} ;$ (b, d) $50 \mu \mathrm{m}$.

Table 1 CNN1 performance assessed on the test set.

Network

\begin{tabular}{|c|c|c|c|c|c|c|c|c|c|c|}
\hline \multirow[b]{2}{*}{ Data augmentation } & & \multicolumn{3}{|c|}{ Accuracy (\%) } & \multicolumn{3}{|c|}{ Sensitivity (\%) } & \multicolumn{3}{|c|}{ Specificity (\%) } \\
\hline & & $M+$ & $M-$ & Other & $M+$ & $M-$ & Other & $M+$ & $M-$ & Other \\
\hline CNN1v1 & No & 98 & 96 & 98 & 94 & 99 & 95 & 100 & 95 & 100 \\
\hline CNN1v2 & Yes & 94 & 91 & 97 & 86 & 86 & 96 & 98 & 92 & 98 \\
\hline
\end{tabular}

$M+$, colonised root sections; $M-$, noncolonised root sections; Other, background and nonroot sections (dusts, air bubbles, etc.).

mispredictions consisted of false positives, such as vesicles or intraradical hyphae predicted in tiles not containing these structures, and confusion between arbuscules and vesicles (Fig. S5a,c).
$\mathrm{CNN} 2$ training on augmented data $(\mathrm{CNN} 2 \mathrm{v} 2)$ triggered the same mispredictions, but also increased the proportion of false negatives (nondetected structures), in particular truncated 
Table 2 CNN2 performance assessed on the test set.

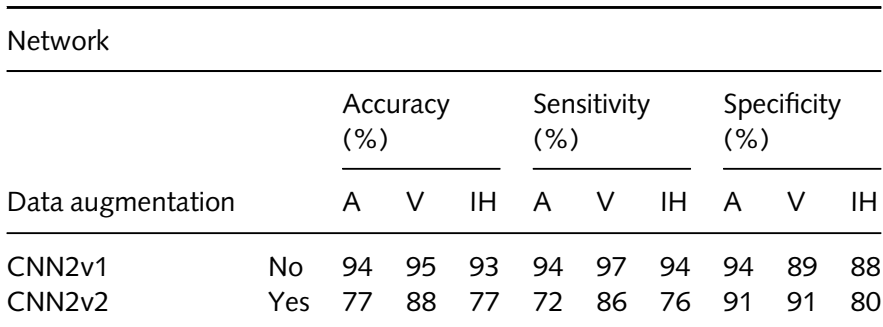

$A$, arbuscules; $I \mathrm{H}$, intraradical hyphae; $V$, vesicles.

arbuscule or vesicle shapes occurring at tile edges, and lowcontrasted intraradical hyphae (Fig. S5b,c).

CNN1 consistently labels fungal colonisation of $N$. benthamiana roots irrespective of the image resolution and can annotate highly dissimilar image datasets, suggesting it may be compatible with a wide range of acquisition devices and staining techniques. CNN2 enables a detailed analysis of fungal hyphal structures, suggesting it may be used to monitor intraradical hyphal structure abundance within host roots. The AMFinder graphical interface allows users to inspect computer predictions and correct mispredictions to work around the limitations of tile-based image segmentation. Thus, AMFinder can robustly identify AM fungal colonisation and intraradical structures.

\section{AMFinder performs consistently on multiple host model species}

A wide range of plants is used in endomycorrhiza research, including legumes and monocot species with various root sizes and morphology. We assessed the suitability of AMFinder pretrained models trained on $N$. benthamiana root images to predict AM fungal colonisation and intraradical hyphal structures on colonised root images from $M$. truncatula ecotype R108 (Fig. 3a), L. japonicus cv Gifu (Fig. 3b), and O. sativa cv Nipponbare (Fig. 3c). The image contrast of ClearSee-treated L. japonicus and M. truncatula roots was similar to $N$. benthamiana. Conversely, large lateral roots of O. sativa showed higher staining background and were more challenging to destain (Fig. 3c). AMFinder correctly identified roots and background in the tested images, with colonised and noncolonised root areas being accurately resolved (Fig. 3a-c), including in cases where colonisation was restricted to inner cortical cell files (Fig. 3b). Overall, CNN1 accuracy reached 97\% (Fig. 3; Table S5). Two mispredicted tiles were manually edited with amfbrowser (Fig. 3b). We then used pre-trained CNN2 to identify and label intraradical hyphal structures (Fig. 3a-c). CNN2 correctly identified arbuscules, vesicles, and intraradical hyphae within roots of the three host plants, reaching an overall accuracy of $90 \%$ (excluding hyphopodia) (Fig. 3; Table S6). Together, these findings suggest AMFinder is compatible with multiple host plants. Roots with high staining background, such as $O$. sativa large lateral roots, may require prolonged destaining for optimal analysis, or specific refinement of AMFinder pretrained models.

\section{AMFinder is compatible with multiple Glomeraceae species}

More than 100 AM fungal species have been reported (Chen $e t$ al., 2018). To determine whether AMFinder pre-trained models are compatible with other fungal mycobionts, we analysed roots from $N$. benthamiana plants colonised with different Glomeraceae species (Fig. 4). Colonised and noncolonised root sections were labelled by CNN1 with an overall accuracy of 97\% (Fig. 4; Table S7). Mispredictions (10 tiles among 160) were manually edited with amfbrowser (Fig. 4). Then, colonised root sections were analysed with CNN2. All colonised root sections contained arbuscules and intraradical hyphae. Vesicles were abundant in sections colonised with either C. claroideum (Fig. 4a) or R. microaggregatum (Fig. 4b), while they were scarce upon colonisation by Funneliformis spp. (Fig. 4c,d). CNN2 correctly identified intraradical hyphal structures in all four species, reaching an overall accuracy of $87 \%$ (excluding hyphopodia) (Fig. 4; Table S8). In particular, $\mathrm{CNN} 2$ predictions mirrored the low abundance of vesicles in Funneliformis-colonised roots (Fig. 4c,d) compared to C. claroideum (Fig. 4a) and R. microaggregatum (Fig. 4b). Thus, AMFinder trained models are compatible with AM fungal species forming similarly shaped intraradical hyphal structures.

\section{AMFinder enables in silico quantification of arbuscular mycorrhizal fungal colonisation dynamics}

We next investigated whether AMFinder could be used to reliably quantify AM colonisation changes of plant roots over time. To that end, we assessed AM fungal colonisation extent on $N$. benthamiana roots harvested after a 4- or 6-wk co-cultivation with $R$. irregularis (Fig. 5). First, we monitored the accumulation of transcripts encoding a $N$. benthamiana ortholog of the mycorrhiza-responsive gene $M t B C P 1 b$ (Parádi et al., 2010) (Fig. $5 \mathrm{a})$ and quantified fungal biomass by monitoring $R$. irregularis EF1a transcript levels (Fig. 5b). Both methods showed a significant, two- to three-fold increase in fungal content at 6 wpi compared to 4 wpi (Fig. 5a,b). Then, using the gridline intersect method (Giovannetti \& Mosse, 1980) we studied the colonisation extent within randomly sampled root fragments (Fig. 5c). Consistent with the molecular analysis, more colonisation was observed at 6 wpi. The analysis of the same samples with AMFinder gave similar results (Fig. 5d). We then tested AMFinder's ability to predict fungal colonisation on low-resolution flatbed scanner pictures of whole root systems (Fig. 5e). Consistent with random sampling and molecular data, AM fungal colonisation levels were significantly higher at $6 \mathrm{wpi}$, although the colonisation extent values were lower than those obtained through random sampling (Fig. 5e). We generated root system maps to gain insights into the distribution of colonised areas (Fig. 5e). We found that while some root sections were entirely colonised, other areas were devoid of colonisation, in particular at 4 wpi (Fig. 5e), suggesting that careful mixing of root fragments is required for quantification based on a fraction of the root systems. We showcased the usefulness of $\mathrm{CNN} 2$ by quantifying intraradical hyphal structures on whole root systems harvested at 2, 3 and 4 wpi (Fig. 5f). Together, these results demonstrate that AMFinder allows 


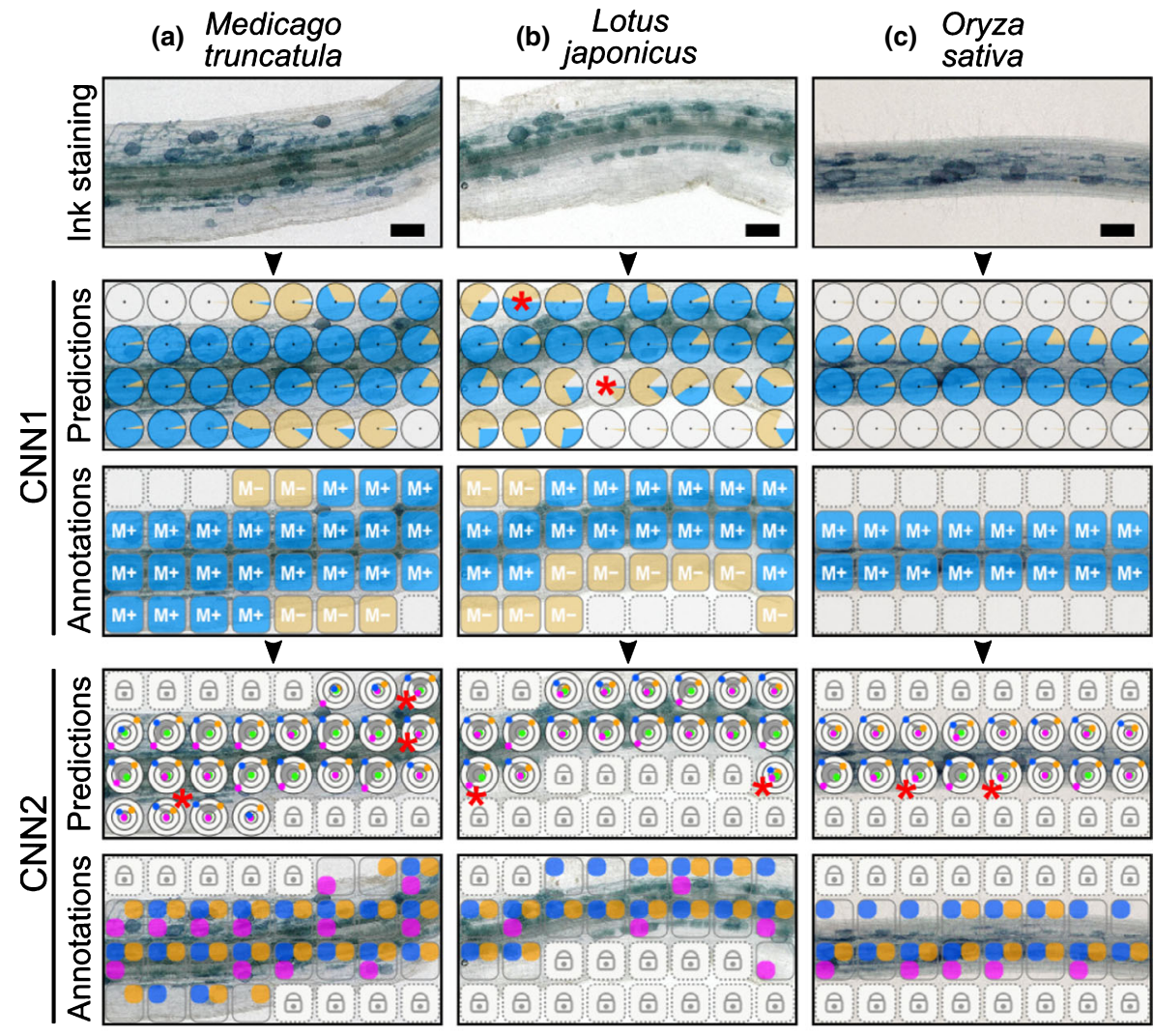

* Mispredictions (edited with amfbrowser)
Fig. 3 AMFinder accurately identifies arbuscular mycorrhizal fungal colonisation and intraradical structures in roots of various host model species. (a-c) Roots from Medicago truncatula (a), Lotus japonicus (b), and Oryza sativa (c) were stained with ink and cleared with ClearSee before analysis with AMFinder. amf mispredictions (red asterisks) were manually edited with amfbrowser. Arrowheads indicate the direction of AMFinder processing. Bars, $50 \mu \mathrm{m}$. for in silico quantification of AM fungal colonisation of plant roots over time, including in whole root systems.

\section{AMFinder accurately quantifies colonisation levels of ram1, str, and smax 1 mutants}

To determine whether AMFinder can identify altered colonisation levels in plant mutants, we quantified colonisation levels of $M$. truncatula roots, either wild-type or carrying the mutations ram1-1 (Gobbato et al., 2012) and str (Zhang et al., 2010) (Fig. 6a-c). Consistent with the phenotypes reported for these mutants, we found that the extent of root colonisation by $R$. irregularis was reduced in ram-1-1 and str backgrounds compared to wild-type (Fig. 6a). Similarly, both mutants showed reduced amounts of arbuscules, vesicles, and intraradical hyphae compared to wild-type, and arbuscules and vesicles were rare in the str mutant (Fig. 6b,c). Microscopic inspection of the colonised roots showed stunted arbuscules in the ram1-1 and str mutant backgrounds compared to wild-type, with a more severe reduction in arbuscule branching in str (Fig. 6c). Next, we investigated whether AMFinder can detect an increased colonisation. We assessed the extent of $R$. irregularis colonisation of $O$. sativa roots in either wild-type plants or those carrying a mutation that abolishes expression of the suppressor of AM symbiosis SMAX1 (Choi et al., 2020). We found that colonisation level was significantly increased in the smax 1 mutant compared to wild-type (Fig. 6d,f). In addition, CNN2 analysis showed that all types of intraradical hyphal structures were more abundant in smax 1 roots (Fig. 6e). Thus, AMfinder accurately detects the AM fungal colonisation phenotypes of well-established plant mutants, suggesting it can adapt to multiple host genetic backgrounds.

\section{Discussion}

We developed the software AMFinder, which uses two CNNs to annotate and quantify AM fungi in plant roots. AMFinder performs consistently well on root images of several model plant and fungal species used in endosymbiosis research. AMFindermediated quantification of AM fungal colonisation gives similar results to those obtained using current standard counting methods. We further show that AMFinder can process whole root systems using low-resolution flatbed scans obtained from an optimised ink-staining protocol which relies on ClearSee as a contrast enhancer. We illustrate the usefulness of this approach to the study of fungal colonisation dynamics over time in wildtype and mutant plants.

AMFinder enables computer-assisted quantification of AM fungal colonisation. A pioneering attempt to automate the quantification of AM fungal colonisation relied on pixel-based image segmentation to determine projected root and fungal surface areas using the proprietary software WINRHIZO, developed by Regent Instruments (Kokkoris et al., 2019). Like AMFinder, this method enabled whole-root-system analyses and showed that quantification based on gridline intersects was generally 

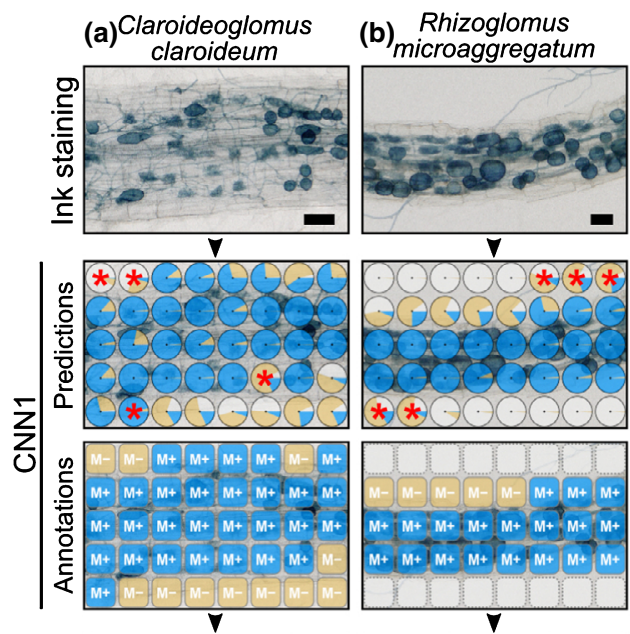

colonisation and intraradical hyphal structures formed by different Glomeraceae species. (a-d) Roots from Nicotiana benthamiana plants growing in the presence of either Claroideog/omus claroideum (a), Rhizoglomus microaggregatum (b), Funneliformis geosporum (c), or

Funneliformis mosseae (d) were stained with ink and cleared with ClearSee before analysis with AMFinder. amf mispredictions (red asterisks) were manually edited with amfbrowser. Arrowheads indicate the direction of AMFinder processing. Bars, $50 \mu \mathrm{m}$.
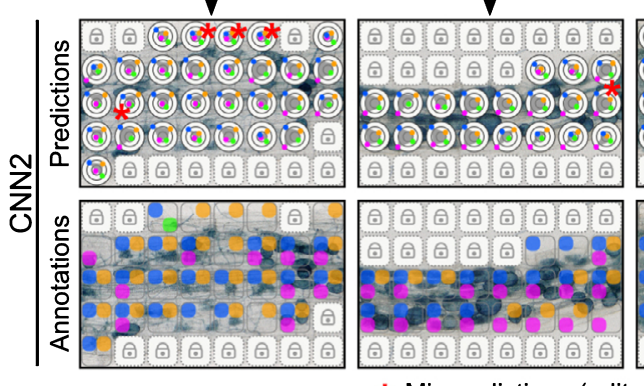

* Mispredictions (edited with amfbrowser) (c) Funneliformis
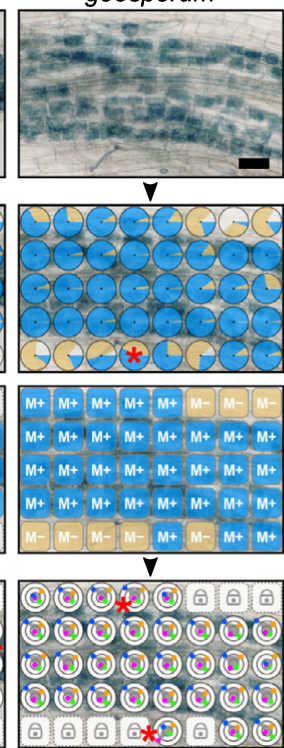

(2) [0]

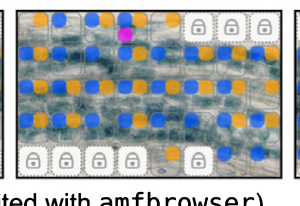

(d) Funneliformis mosseae
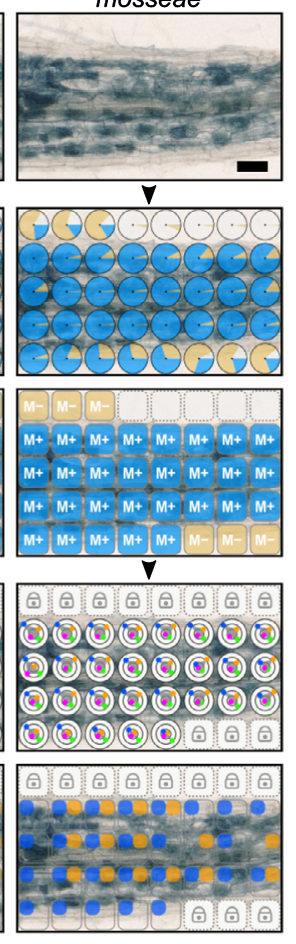

overestimated (Kokkoris et al., 2019). In contrast to pixel-based methods, CNNs are trainable and can thus adapt to a wide range of images, including environmental samples colonised with both $\mathrm{AM}$ and nonAM fungal species. Furthermore, $\mathrm{CNN}$ s further enable the quantification of different types of intraradical hyphal structures, which are important parameters of plant mutant phenotypes. Thus, deep learning ensures AMFinder versatility and enables a more detailed analysis of mycorrhizal phenotypes.

AMFinder's design adequately addresses limitations arising from its computer vision approach while still enabling a low- to medium-throughput workflow. Specifically, we implemented a semi-automatic pipeline that requires user supervision of computer predictions. High-throughput AMFinder analyses, such as large-scale field experiments, would require the entire prediction pipeline to be fully automatic, including the conversion to annotations. However, input image parameters can influence pre-trained model accuracy and may require user adjustments. Automatic analyses assuming image suitability without quality control may overestimate CNN model accuracy. CNN2 predictions on mispredicted $\mathrm{M}+$ tiles without intraradical hyphal structures have not been investigated in this study. Besides, this AMFinder implementation does not discriminate between multiple root types. As a result, image data from experiments relying on crude inoculum (Habte \& Byappanhalli, 1998) or nurse root systems such as chives (Demchenko et al., 2004) as an inoculation method may pose problems when contaminating root fragments remain in root images. To that end, AMFinder offers manual curation of predictions to avoid systematic introduction of errors.
We used an uncertainty sampling-like bootstrap method, wherein $\mathrm{CNNs}$ are trained on a small amount of manually annotated images, and then the user is prompted to label further training images which the model is unsure about, for rapid creation of a large training dataset. Despite misannotations in the resulting training dataset, AMFinder trained networks showed high accuracy on a large set of images featuring different plant and fungal species, confirming the suitability of this strategy. Further improvements could possibly be achieved using an active learning approach (Shen et al., 2018).

We trained and tested AMFinder on ink-vinegar stained $N$. benthamiana roots. Ink-vinegar is an inexpensive, nontoxic fungal staining method compatible with various plant and mycobiont species (Vierheilig et al., 1998). Thus, pre-trained CNNs generated from ink-stained roots ensure immediate workability of AMFinder for most endosymbiosis host systems without the need to generate manually annotated training datasets. We augmented AMFinder capabilities by implementing optional data augmentation consisting of image flipping and colour alteration. Data augmentation is performed on the fly during training and does not affect AMFinder performance. We showed that enabling such image transformations during training allows for accurate labelling of images with altered hue, intensity, and background colours. If needed, AMFinder can be trained with datasets obtained using other dyes and fluorophores for fungal staining (Vierheilig et al., 2005) or for the annotation of other tissues colonised by fungi such as liverwort thalli (Ligrone et al., 2007; Carella \& Schornack, 2018; Kobae et al., 2019). Computerintensive computations required for ab initio training can be 

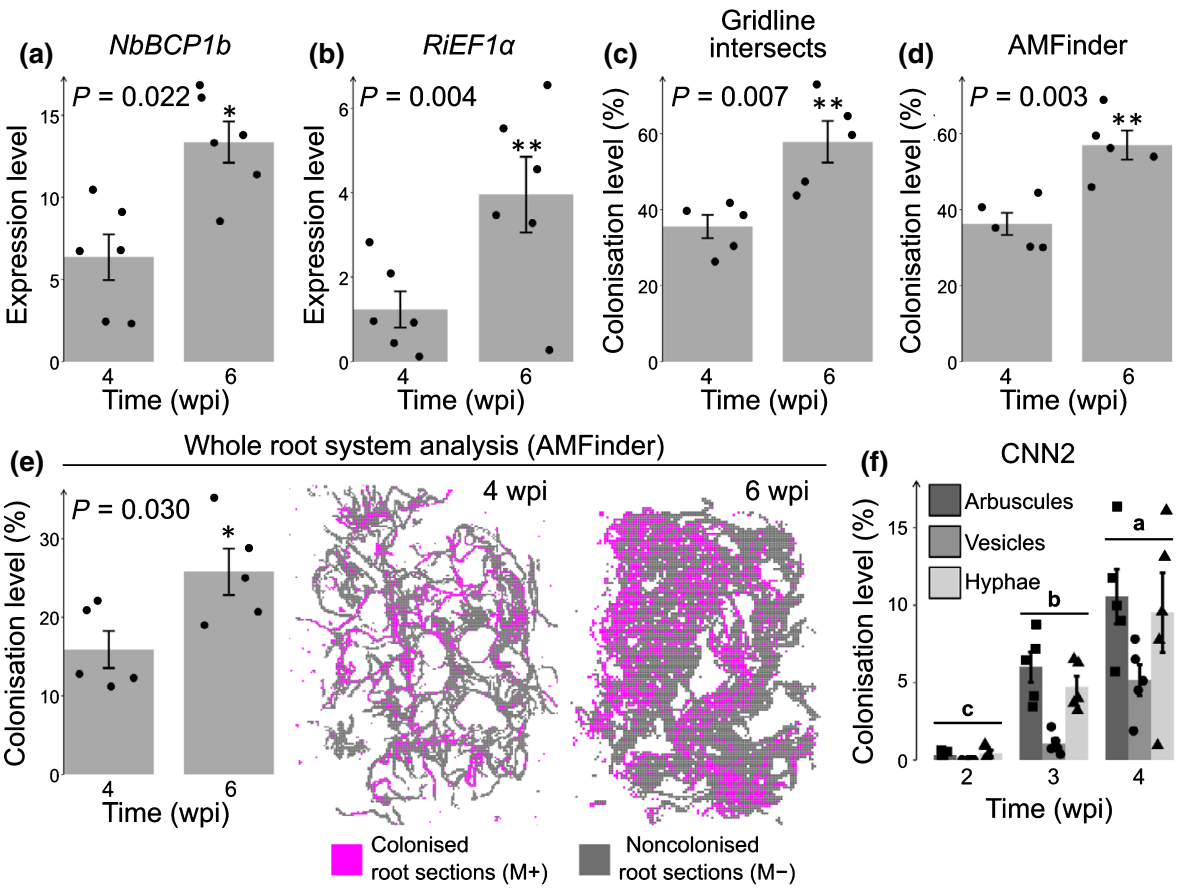

Fig. 5 Computer vision enables quantification of arbuscular mycorrhizal (AM) fungal colonization on whole root systems. (a-f) Nicotiana benthamiana plants were inoculated with Rhizophagus irregularis. Colonisation levels were determined 4 and 6 wk post-inoculation (wpi). (a, b) Quantification of transcripts corresponding to the $N$. benthamiana mycorrhizal marker gene Blue Copper Protein 1 (BCP1) (a) and the Rhizophagus irregularis Elongation Factor $1 \alpha(E F 1 \alpha)($ b). Data are expressed relative to both $N$. benthamiana $L 23$ and $F$-box reference genes. (c, d) Quantification of AM fungal colonization on ink-stained root pictures using the gridline intersect method (c), or AMFinder (d). (e) Quantification of AM fungal colonisation on whole root systems using AMFinder, and representative images of computer-generated maps featuring colonized ( $M+$, magenta) and noncolonized ( $M-$, grey) root areas at 4 and 6 wpi. (f) Intraradical hyphal structures were quantified on whole root systems at 2, 3, and 4 wpi using neural network 2 (CNN2). Dots correspond to biological replicates. Bars represent SE. Statistical significance was assessed using Student's $t$-test $(*, P<0.05 ; * *, P<0.01)$. Squares, triangles, and circles indicate actual values. Lowercase letters indicate statistical grouping.

avoided by refining the existing pre-trained networks. Thus, AMFinder is highly versatile and can be adapted for the study of many aspects of fungal colonisation; it may also be of interest to researchers of pathogenic fungi.

AMFinder sensitivity is best on vesicles, likely because AM fungal vesicles are fairly invariant, globular-shaped structures surrounded by a thick, multi-layered wall (Jabaji-Hare et al., 1990), resulting in high contrast signals within the surrounding plant tissues. By contrast, the arbuscular shape is more diverse, with branching extent and cell volume occupancy increasing during the initial development stages (Toth \& Miller, 1984) and a size that is ultimately defined by host cell size. Intraradical hyphae show different diameters, orientations, and staining intensities, and occasionally overlay other intraradical structures. The limited pixel information of a single tile may not always discriminate between intraradical and extraradical hyphae. An approach using information from a wider area of the original image, rather than treating each tile in isolation, may help to address this issue. In particular, it would be interesting to apply deep learning image segmentation techniques (Ghosh et al., 2019) to this problem, as researchers have often found success with this approach in other types of biological imaging. Another possible issue is that CNNs do not retain relative spatial information (Patrick et al., 2019). Solutions to overcome this limitation include the combination of CNNs and multi-layer perceptrons (Haldekar et al., 2017), and capsule networks (CapsNets) (Sabour et al., 2017; Patrick et al., 2019). Future work will explore the usefulness of such approaches to achieve even higher prediction accuracy.

Obtaining contrasted fungal structures within root tissues is pivotal for accurate AMFinder predictions. The first report of ink-vinegar staining of AM fungi suggests that black and blue inks allow for high-contrast images in at least four plant species (Vierheilig et al., 1998). Background destaining in tap water with a few vinegar droplets requires at least $20 \mathrm{~min}$ incubation and is only effective against excess ink (Vierheilig et al., 1998). By contrast, ClearSee treatment works in seconds and allows for both destaining and clearing (Kurihara et al., 2015). Such a feature is of particular interest for thick or pigmented roots, and soil samples. Also, ClearSee preserves fluorescence (Kurihara et al., 2015) and is thus compatible with immunohistochemical fungal labelling techniques such as wheat germ agglutinin-fluorophore conjugates (Bonfante-Fasolo et al., 1990).

AMFinder can improve the robustness and reproducibility of AM fungal quantification. In the gridline-intersect method, gridlines have been primarily used as guides for the systematic selection of observation points (Giovannetti \& Mosse, 1980), and the distance between adjacent lines has been studied to estimate the total root length, but not to improve quantification accuracy (Newman, 1966; Marsh, 1971; Giovannetti \& Mosse, 1980). As a result, a low number of root fragments is considered prejudicial to 

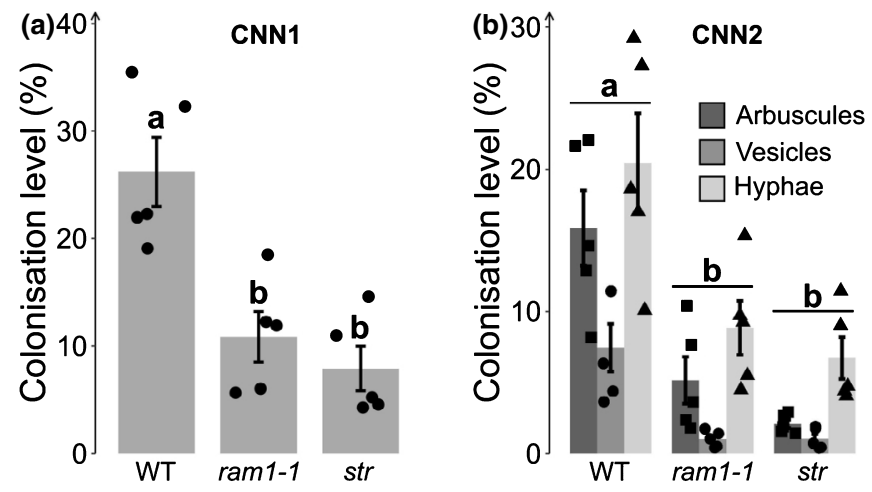

(c)

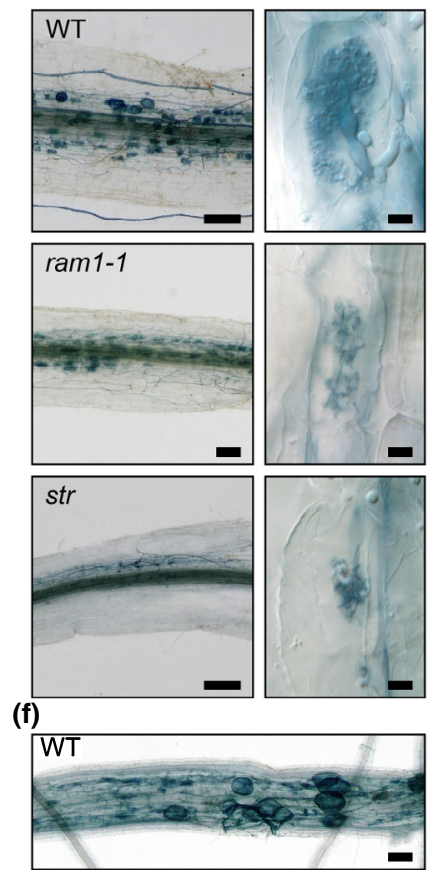

(d)
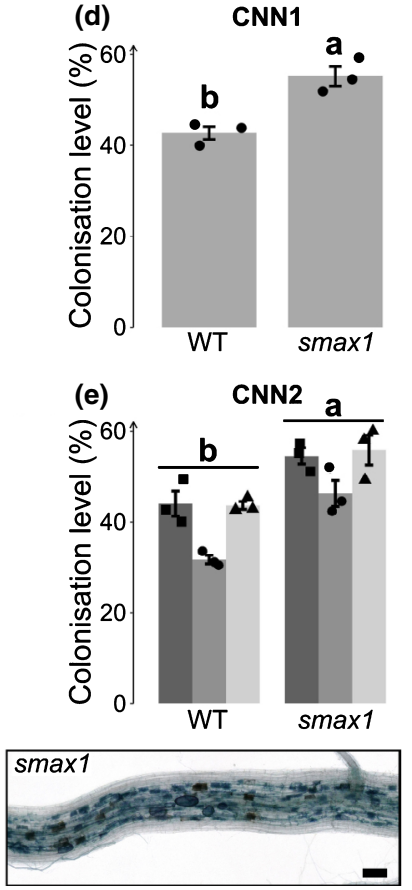

Fig. 6 AMFinder accurately quantifies altered colonisation levels of ram1, str, and smax 1 mutants. (a-c) Medicago truncatula A17 (wild-type), ram1-1, and str were grown on sand for $6 \mathrm{wk}$ in the presence of Rhizophagus irregularis. Bar plots show overall colonisation level obtained using neural network 1 (CNN1) (a) and a detailed analysis of root content in arbuscules, vesicles, and intraradical hyphae obtained by CNN2 analysis (b). Representative images of colonised roots and arbuscule shape (c). (df) Oryza sativa wild-type and smax 1 were grown on sand for 6 wk in the presence of $R$. irregularis. Bar plots show overall colonisation levels (d) and quantification of intraradical hyphal structures (e). (f) Representative images of colonised large lateral roots. Statistical significance was assessed using ANOVA and Tukey's HSD $(P<0.05)$. Letters indicate statistical groupings. Bars represent the SE. Bars: (c) $100 \mu \mathrm{m}$ (left panels), $10 \mu \mathrm{m}$ (right panels); (f) $100 \mu \mathrm{m}$.

quantification accuracy (Giovannetti \& Mosse, 1980). Also, the shape of the area surrounding the grid-root intersection used for visual scoring has not been formally described and may account for variations between experimenters. By contrast, AMFinder analyses well-defined tiles, and tile size can adjust to image resolution without impairing prediction accuracy. Intraradical hyphal structures cannot be identified from flatbed scans due to the limited resolution of the images produced. However, machine learning-based algorithms have been recently developed to achieve data-driven image super-resolution (Park et al., 2003; Wang et al., 2019). In contrast to standard image interpolation techniques, super-resolution algorithms predict missing details by learning common patterns from training datasets. Whether such algorithms can enable a detailed analysis of AM fungal hyphal structures from flatbed scans could be explored in future AMFinder developments.

\section{Conclusions}

We have demonstrated that AMFinder adapts to different plant and fungal species, fungal staining methods, and acquisition devices. Its design ensures user control over the annotation process and facilitates data visualisation in the context of the root images. As such, it improves documentation and reproducibility of AM fungal colonisation analyses.

\section{Acknowledgements}

We thank Raymond Wightman (SLCU, Cambridge) for assistance with digital microscopy. We are grateful to Johannes Stuttmann (Martin-Luther-Universität Halle-Wittenberg, Germany) for critical comments on the manuscript. We thank Colleen Drapek (SLCU, Cambridge) for providing us with Funneliformis mosseae inoculum. This work was funded by the Gatsby Charitable Foundation (GAT3395/GLD), by the European Research Council (ERC2014-STG, H2020 and 637537), and by the Royal Society (UF110073 and UF160413). CT is supported by a Junior Research Fellowship at Gonville \& Caius College, Cambridge, and acknowledges support by the STFC consolidated grant ST/P000681/1.

\section{Author contributions}

EE developed the software, conceived the experimental strategy, conducted experiments, acquired data, analysed data, and wrote the manuscript. CT developed the software and analysed data. AM conceived the original idea, conducted experiments, and acquired data. LS, TY and AG conducted experiments and acquired data. EKS and CQ contributed material. SS acquired funding, conceived the experimental strategy, analysed data, and wrote the manuscript. All authors have read and approved the manuscript.

\section{ORCID}

Edouard Evangelisti (iD https://orcid.org/0000-0002-7218-7850

Aleksandr Gavrin (D) https://orcid.org/0000-0003-0179-8491

Clément Quan (iD https://orcid.org/0000-0002-6531-4903

Sebastian Schornack (D) https://orcid.org/0000-0002-7836-5881

Liron Shenhav (iD) https://orcid.org/0000-0003-1961-4187

Carl Turner (D) https://orcid.org/0000-0001-7202-5731

Temur Yunusov (D) https://orcid.org/0000-0001-9936-4354

\section{Data availability}

AMFinder is released under the terms of the open-source MIT license (https://opensource.org/licenses/MIT) allowing 
unrestricted usage. Source code, pre-trained models and detailed installation instructions are available on the AMFinder GitHub webpage (https://github.com/SchornacklabSLCU/amfinder.git). Training datasets are available on Zenodo (https://doi.org/10. 5281/zenodo.5118948).

\section{References}

Abadi M, Agarwal A, Barham P, Brevdo E, Chen Z, Citro C, Corrado GS, Davis A, Dean J, Devin M et al. 2016. TensorFlow: large-scale machine learning on heterogeneous distributed systems. arXiv: 1603.04467.

Bally J, Jung H, Mortimer C, Naim F, Philips JG, Hellens R, Bombarely A, Goodin MM, Waterhouse PM. 2018. The rise and rise of Nicotiana benthamiana: a plant for all reasons. Annual Review of Phytopathology 56: 405426.

Bojarski M, Del Testa D, Dworakowski D, Firner B, Flepp B, Goyal P, Jackel LD, Monfort M, Muller U, Zhang J et al. 2016. End to end learning for selfdriving cars. arXiv: 1604.07316 .

Bonfante-Fasolo P, Faccio A, Perotto S, Schubert A. 1990. Correlation between chitin distribution and cell wall morphology in the mycorrhizal fungus Glomus versiforme. Mycological Research 94: 157-165.

Brundrett MC. 2007. Scientific approaches to Australian temperate terrestrial orchid conservation. Australian Journal of Botany 55: 293.

Brundrett MC, Tedersoo L. 2018. Evolutionary history of mycorrhizal symbioses and global host plant diversity. New Phytologist 220: 1108-1115.

Carella P, Schornack S. 2018. Manipulation of bryophyte hosts by pathogenic and symbiotic microbes. Plant and Cell Physiology 59: 651-660.

Chen M, Arato M, Borghi L, Nouri E, Reinhardt D. 2018. Beneficial services of arbuscular mycorrhizal fungi - from ecology to application. Frontiers in Plant Science 9: 1270 .

Choi J, Lee T, Cho J, Servante EK, Pucker B, Summers W, Bowden S, Rahimi M, An K, An G et al. 2020. The negative regulator SMAX1 controls mycorrhizal symbiosis and strigolactone biosynthesis in rice. Nature Communications 11: 1-13.

Chollet F. 2015. Keras, GitHub. [WWW document] URL https://github.com/fc hollet/keras [accessed 1 July 2021].

Demchenko K, Winzer T, Stougaard J, Parniske M, Pawlowski K. 2004. Distinct roles of Lotus japonicus SYMRK and SYM15 in root colonization and arbuscule formation. New Phytologist 163: 381-392.

Dhillon A, Verma GK. 2020. Convolutional neural network: a review of models, methodologies and applications to object detection. Progress in Artificial Intelligence 9: 85-112.

Dickson S. 2004. The Arum-Paris continuum of mycorrhizal symbioses. New Phytologist 163: 187-200.

Ferentinos KP. 2018. Deep learning models for plant disease detection and diagnosis. Computers and Electronics in Agriculture 145: 311-318.

Ghosh S, Das N, Das I, Maulik U. 2019. Understanding deep learning techniques for image segmentation. ACM Computing Surveys 52: 1-35.

Giovannetti M, Mosse B. 1980. An evaluation of techniques for measuring vesicular arbuscular mycorrhizal infection in roots. New Phytologist 84: 489500.

Gobbato E, Marsh J, Vernié T, Wang E, Maillet F, Kim J, Miller J, Sun J, Bano $S$, Ratet $\mathrm{P}$ et al. 2012. A GRAS-type transcription factor with a specific function in mycorrhizal signaling. Current Biology 22: 2236-2241.

Gordon-Rodriguez E, Loaiza-Ganem G, Pleiss G, Cunningham JP. 2020. Uses and abuses of the cross-entropy loss: case studies in modern deep learning. arXiv: 2011.05231.

Habte M, Byappanhalli BN. 1998. Influence of pre-storage drying conditions and duration of storage on the effectiveness of root inoculum of Glomus aggregatum. Journal of Plant Nutrition 21: 1375-1389.

Haldekar M, Ganesan A, Oates T. 2017. Identifying spatial relations in images using convolutional neural networks. arXiv: 1706.04215 .

Harrison MJ, Dewbre GR, Liu J. 2002. A phosphate transporter from Medicago truncatula involved in the acquisition of phosphate released by arbuscular mycorrhizal fungi. Plant Cell 14: 2413-2429.
Helber N, Requena N. 2008. Expression of the fluorescence markers DsRed and GFP fused to a nuclear localization signal in the arbuscular mycorrhizal fungus Glomus intraradices. New Phytologist 177: 537-548.

Hewitt EJ. 1966. Sand and water culture methods used in the study of plant nutrition, $2^{\text {nd }}$ edn. Farnham Royal, UK: Commonwealth Agricultural Bureaux.

Hohnjec N, Vieweg MF, Pühler A, Becker A, Küster H. 2005. Overlaps in the transcriptional profiles of Medicago truncatula roots inoculated with two different Glomus fungi provide insights into the genetic program activated during arbuscular mycorrhiza. Plant Physiology 137: 1283-1301.

Hulse JD. 2018. Review of comprehensive staining techniques used to differentiate arbuscular mycorrhizal fungi from plant root tissues. Acta Scientific Agriculture 2: 39-44.

Jabaji-Hare SH, Theiren J, Charest PM. 1990. High resolution cytochemical study of the vesicular-arbuscular mycorrhizal association, Glomus clarum $\mathrm{x}$ Allium porrum. New Phytologist 114: 481-496.

Javot H, Penmetsa RV, Terzaghi N, Cook DR, Harrison MJ. 2007. A Medicago truncatula phosphate transporter indispensable for the arbuscular mycorrhizal symbiosis. Proceedings of the National Academy of Sciences, USA 104: 17201725

Kingma DP, Ba JL. 2015. Adam: a method for stochastic optimization. arXiv: 1412.6980.

Kobae Y, Ohtomo R, Morimoto S, Sato D, Nakagawa T, Oka N, Sato S. 2019. Isolation of native arbuscular mycorrhizal fungi within young thalli of the liverwort Marchantia paleacea. Plants 8: 142.

Kokkoris V, Pogiatzis A, Hart MM. 2019. Contrasting common measures of arbuscular mycorrhizal fungal root colonization. Journal of Microbiological Methods 167: 105727.

Kurihara D, Mizuta Y, Sato Y, Higashiyama T. 2015. ClearSee: a rapid optical clearing reagent for whole-plant fluorescence imaging. Development 142: 4168 4179.

LeCun Y, Bottou L, Bengio Y, Haffner P. 1998. Gradient-based learning applied to document recognition. Proceedings of the IEEE 86: 2278-2323.

Leroy X, Doligez D, Frisch A, Garrigue J, Rémy D, Vouillon J. 2020. The OCaml system, release 4.11 documentation and user's manual. Technical report, Inria. [WWW document] URL https://hal.inria.fr/hal-00930213v7 [accessed 1 July 2021].

Ligrone R, Carafa A, Lumini E, Bianciotto V, Bonfante P, Duckett JG. 2007. Glomeromycotean associations in liverworts: a molecular, cellular, and taxonomic analysis. American Journal of Botany 94: 1756-1777.

Liu D, Shi L, Han C, Yu J, Li D, Zhang Y. 2012. Validation of reference genes for gene expression studies in virus-infected Nicotiana benthamiana using quantitative real-time PCR. PLoS ONE 7: e46451.

Liu Y, Tang F, Zhou D, Meng Y, Dong W. 2017. Flower classification via convolutional neural network. In: Proceedings - 2016 IEEE International Conference on Functional-Structural Plant Growth Modeling, Simulation, Visualization and Applications, FSPMA 2016. Manhattan, NY, USA: Institute of Electrical and Electronics Engineers, 110-116.

Luginbuehl LH, Oldroyd GED. 2017. Understanding the arbuscule at the heart of endomycorrhizal symbioses in plants. Current Biology 27: R952-R963.

Maeda D, Ashida K, Iguchi K, Chechetka SA, Hijikata A, Okusako Y, Deguchi Y, Izui K, Hata S. 2006. Knockdown of an arbuscular mycorrhiza-inducible phosphate transporter gene of Lotus japonicus suppresses mutualistic symbiosis. Plant and Cell Physiology 47: 807-817.

Marsh B. 1971. Measurement of length in random arrangements of lines. The Journal of Applied Ecology 8: 265.

Matsugu M, Mori K, Mitari Y, Kaneda Y. 2003. Subject independent facial expression recognition with robust face detection using a convolutional neural network. Neural Networks 16: 555-559.

Mohanty SP, Hughes DP, Salathé M. 2016. Using deep learning for imagebased plant disease detection. Frontiers in Plant Science 7: 1419.

Montero H, Choi J, Paszkowski U. 2019. Arbuscular mycorrhizal phenotyping: the dos and don'ts. New Phytologist 221: 1182-1186.

Newman EI. 1966. A method of estimating the total length of root in a sample. The Journal of Applied Ecology 3: 139.

O’Mahony N, Campbell S, Carvalho A, Harapanahalli S, Hernandez GV, Krpalkova L, Riordan D, Walsh J. 2020. Deep learning vs. traditional 
computer vision. In: Arai K, Kapoor S, eds. Advances in intelligent systems and computing. Cham, Switzerland: Springer International Publishing, 128-144.

Parádi I, Van Tuinen D, Morandi D, Ochatt S, Robert F, Jacas L, Dumas-

Gaudot E. 2010. Transcription of two blue copper-binding protein isogenes is highly correlated with arbuscular mycorrhizal development in Medicago truncatula. Molecular Plant-Microbe Interactions 23: 1175-1183.

Park SC, Park MK, Kang MG. 2003. Super-resolution image reconstruction: a technical overview. IEEE Signal Processing Magazine 20: 21-36.

Patrick M, Adekoya F, Mighty A, Edward B. 2019. Capsule networks - a survey. Journal of King Saud University - Computer and Information Sciences. doi: 10. 1016/j.jksuci.2019.09.014.

van Rossum G, Drake FL. 2009. Python 3 reference manual. Scotts Valley, CA, USA: CreateSpace.

Sabour S, Frosst N, Hinton GE. 2017. Dynamic routing between capsules. arXiv: 1710.09829 .

Schüßler A, Schwarzott D, Walker C. 2001. A new fungal phylum, the Glomeromycota: phylogeny and evolution. Mycological Research 105: 14131421.

Shen Y, Yun H, Lipton ZC, Kronrod Y, Anandkumar A. 2018. Deep active learning for named entity recognition. arXiv: 1707.05928.

Spatafora JW, Chang Y, Benny GL, Lazarus K, Smith ME, Berbee ML, Bonito G, Corradi N, Grigoriev I, Gryganskyi A et al. 2016. A phylum-level phylogenetic classification of zygomycete fungi based on genome-scale data. Mycologia 108: 1028-1046.

Srinath KR. 2017. Python-the fastest growing programming language. International Research Journal of Engineering and Technology 4: 354-357.

Sun XG, Tang M. 2012. Comparison of four routinely used methods for assessing root colonization by arbuscular mycorrhizal fungi. Botany-Botanique 90: 1073-1083.

Sun Y, Liu Y, Wang G, Zhang H. 2017. Deep learning for plant identification in natural environment. Computational Intelligence and Neuroscience 2017: 7361042.

Szarvas M, Yoshizawa A, Yamamoto M, Ogata J. 2005. Pedestrian detection with convolutional neural networks. In: IEEE Intelligent Vehicles Symposium, Proceedings. 224-229. doi: 10.1109/IVS.2005.1505106.

Takeda N, Sato S, Asamizu E, Tabata S, Parniske M. 2009. Apoplastic plant subtilases support arbuscular mycorrhiza development in Lotus japonicus. The Plant Journal 58: 766-777.

Thapa R, Zhang K, Snavely N, Belongie S, Khan A. 2020. The Plant Pathology Challenge 2020 data set to classify foliar disease of apples. Applications in Plant Sciences 8: e11390.

Toth R, Miller RM. 1984. Dynamics of arbuscule development and degeneration in a Zea mays mycorrhiza. American Journal of Botany 71: 449.

Vandesompele J, De Preter K, Pattyn F, Poppe B, Van Roy N, De Paepe A, Speleman F. 2002. Accurate normalization of real-time quantitative RT-PCR data by geometric averaging of multiple internal control genes. Genome Biology 3: research0034.1.

Vierheilig H, Coughlan AP, Wyss U, Piché Y. 1998. Ink and vinegar, a simple staining technique for arbuscular-mycorrhizal fungi. Applied and Environmental Microbiology 64: 5004-5007.

Vierheilig H, Schweiger P, Brundrett M. 2005. An overview of methods for the detection and observation of arbuscular mycorrhizal fungi in roots. Physiologia Plantarum 125: 393-404.

Volpe V, Giovannetti M, Sun XG, Fiorilli V, Bonfante P. 2016. The phosphate transporters LjPT4 and MtPT4 mediate early root responses to phosphate status in non mycorrhizal roots. Plant, Cell \& Environment 39: 660-671.

Voulodimos A, Doulamis N, Doulamis A, Protopapadakis E. 2018. Deep learning for computer vision: a brief review. Computational Intelligence and Neuroscience 2018: 7068349.
Wang Z, Chen J, Hoi SCH. 2019. Deep learning for image super-resolution: a survey. arXiv: 1902.06068.

Yamashita R, Nishio M, Do RKG, Togashi K. 2018. Convolutional neural networks: an overview and application in radiology. Insights into Imaging 9 : 611-629.

You FM, Huo N, Gu YQ, Luo MC, Ma Y, Hane D, Lazo GR, Dvorak J, Anderson OD. 2008. BatchPrimer3: a high throughput web application for PCR and sequencing primer design. BMC Bioinformatics 9: 1-13.

Zhang Q, Blaylock LA, Harrison MJ. 2010. Two Medicago truncatula half-ABC transporters are essential for arbuscule development in arbuscular mycorrhizal symbiosis. Plant Cell 22: 1483-1497.

\section{Supporting Information}

Additional Supporting Information may be found online in the Supporting Information section at the end of the article.

Fig. S1 ClearSee enhances the contrast of ink-stained roots.

Fig. S2 Optimisation of flatbed scanner resolution for inkstained root imaging.

Fig. S3 Schematic representation of AMFinder ConvNet architecture.

Fig. S4 Analysis of CNN1 mispredictions.

Fig. S5 Analysis of CNN2 mispredictions.

Table S1 Primers used in this study.

Table S2 CNN1 training dataset.

Table S3 CNN2 training dataset.

Table S4 Test dataset.

Table S5 CNN1 performance on various host plants.

Table S6 CNN2 performance on various host plants.

Table S7 CNN1 performance on various arbuscular mycorrhizal fungi.

Table S8 CNN2 performance on various arbuscular mycorrhizal fungi.

Please note: Wiley Blackwell are not responsible for the content or functionality of any Supporting Information supplied by the authors. Any queries (other than missing material) should be directed to the New Phytologist Central Office. 